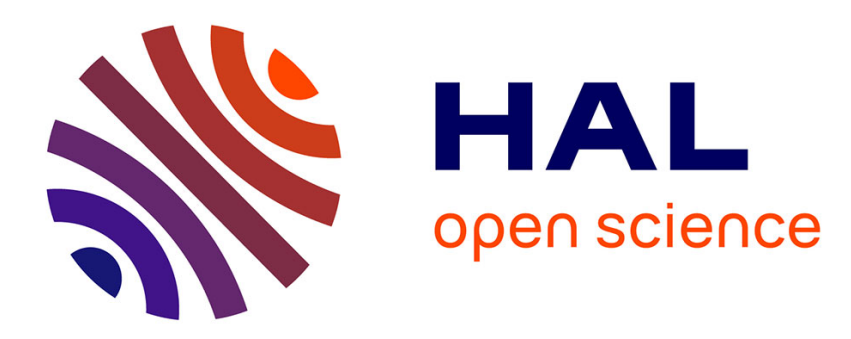

\title{
Pour une histoire sociale de l'asile politique en France
}

Karen Akoka, Alexis Spire

\section{To cite this version:}

Karen Akoka, Alexis Spire. Pour une histoire sociale de l'asile politique en France. Pouvoirs - Revue française d'études constitutionnelles et politiques, 2013, 144 (1), 10.3917/pouv.144.0067 . hal01911841

\section{HAL Id: hal-01911841 \\ https://hal.science/hal-01911841}

Submitted on 3 Nov 2018

HAL is a multi-disciplinary open access archive for the deposit and dissemination of scientific research documents, whether they are published or not. The documents may come from teaching and research institutions in France or abroad, or from public or private research centers.
L'archive ouverte pluridisciplinaire $\mathbf{H A L}$, est destinée au dépôt et à la diffusion de documents scientifiques de niveau recherche, publiés ou non, émanant des établissements d'enseignement et de recherche français ou étrangers, des laboratoires publics ou privés. 


\title{
POUR UNE HISTOIRE SOCIALE DE L'ASILE POLITIQUE EN FRANCE
}

\author{
Karen Akoka et Alexis Spire
}

Le Seuil | Pouvoirs

$2013 / 1-n^{\circ} 144$
pages 67 à 77

ISSN 0152-0768

Article disponible en ligne à l'adresse:

http://www.cairn.info/revue-pouvoirs-2013-1-page-67.htm

Pour citer cet article :

Akoka Karen et Spire Alexis, «Pour une histoire sociale de l'asile politique en France »,

Pouvoirs, 2013/1 n¹44, p. 67-77. DOI : 10.3917/pouv.144.0067

Distribution électronique Cairn.info pour Le Seuil.

(c) Le Seuil. Tous droits réservés pour tous pays.

La reproduction ou représentation de cet article, notamment par photocopie, n'est autorisée que dans les limites des conditions générales d'utilisation du site ou, le cas échéant, des conditions générales de la licence souscrite par votre établissement. Toute autre reproduction ou représentation, en tout ou partie, sous quelque forme et de quelque manière que ce soit, est interdite sauf accord préalable et écrit de l'éditeur, en dehors des cas prévus par la législation en vigueur en France. II est précisé que son stockage dans une base de données est également interdit. 


\section{KAREN AKOKA ET Alexis SPIRE}

\section{POUR UNE HISTOIRE SOCIALE DE L'ASILE POLITIQUE EN FRANCE}

histoire contemporaine de l'asile politique en France a souvent été écrite comme une succession de textes et d'événements conduisant à l'instauration d'un statut pour les réfugiés. Si le principe du droit d'asile a d'abord été énoncé par les révolutionnaires de 1793, ce n'est qu'après la Seconde Guerre mondiale qu'il est entériné dans le Préambule de la Constitution. Il acquiert ensuite un statut international avec l'adoption de la convention de Genève en 1951, ratifiée par la France trois ans plus tard; en 1967, son champ d'application est étendu aux victimes de tous les événements survenus après 1951 et hors d'Europe.

À cette chronologie juridique et institutionnelle, on voudrait substituer une histoire sociale de l'asile politique qui rende compte non pas des règles de droit et des dispositifs régissant le traitement des réfugiés, mais plutôt des pratiques et des trajectoires des agents qui ont eu à les mettre en œuvre. La relation que l'on voudrait explorer entre le profil social des agents recrutés, leurs pratiques à l'égard des réfugiés et les priorités des gouvernants, pourrait constituer une autre manière d'écrire l'histoire de l'État et de son rapport à l'asile politique ${ }^{1}$.

1. Pour plus de détails sur ces profils et ces pratiques voir Karen Akoka, L'Administration de l'asile. La fabrique du réfugié par l'OFPRA (1952-1990), Thèse pour le doctorat de sociologie, décembre 2012. 
UNE GESTION CONSULAIRE

DE L'ASILE POLITIQUE (I945-I980)

À la Libération, le terme de «réfugiés » regroupe, d'une part, les personnes déplacées par la guerre qui arrivent en France, pour la plupart en provenance d'Allemagne, et, d'autre part, certaines nationalités très spécifiques, les Russes, les Arméniens et les Espagnols qui sont pris en charge par des Offices de réfugiés. Le débat qui traverse alors l'appareil d'État est de savoir si la reconnaissance du statut de réfugié peut être déléguée à une autorité indépendante ou si elle doit être assumée par des agents de l'administration française.

Du point de vue du séjour, l'ordonnance du 2 novembre 1945 ne distingue pas les réfugiés des autres étrangers ${ }^{2}$. Plus globalement les années cinquante et soixante sont marquées par une certaine porosité entre traitement de l'asile et appel à la main-d'œuvre étrangère, même si, dès 1946, le gouvernement confie à l'Organisation internationale pour les réfugiés (OIR) le pouvoir de reconnaître la qualité de réfugié à ceux qui fuient les persécutions. À la fin de l'année 1947, cette noningérence est remise en cause: le déclenchement des grèves de l'automne alimente un climat de méfiance à l'encontre des communistes, ce qui rejaillit sur les Espagnols qui demandent le statut de réfugié. D’un côté, les préfectures dénoncent leur activisme politique et, de l'autre, le ministère du Travail exige qu'ils soient plus strictement contrôlés compte tenu de la progression du chômage ${ }^{3}$. Le ministère de l'Intérieur conteste la légitimité des agents espagnols mandatés par l'orr, qui sont pour la plupart d'anciens réfugiés, et demande leur remplacement par des ressortissants français qui « offriraient toutes les garanties requises». En juillet 1949, tout le personnel de la délégation de Perpignan est ainsi remplacé par des agents des Renseignements généraux, ce qui induit une chute du nombre d'Espagnols admis à séjourner en France. Cet épisode préfigure en fait la controverse qui, à la veille de la ratification de la convention de Genève, oppose d'un côté les tenants d'une protection des réfugiés confiée à un organisme international indépendant de toute tutelle étatique, et de l'autre les partisans d'un Office national contrôlé par le gouvernement du pays de résidence. Si la définition du réfugié

2. Cf. Patrick Weil, «Racisme et discrimination dans la politique française de l'immigration 1938-1945/1974-1995 ", Vingtième Siècle, n 47, juillet-septembre 1995, p. 95.

3. Circulaire confidentielle du ministère du Travail du 3 décembre 1948, Archives nationales (AN) F7 16075. 
retenue finalement dans la convention de Genève de 1951 se veut universelle, le texte laisse aux États une grande marge de manœuvre quant aux conditions de son application. En France, la loi sur la création de l'Office français de protection des réfugiés et apatrides (OFPRA) votée en juillet 1952 peut apparaître comme le signe d'une préférence pour une bureaucratie nationale. Mais, en pratique, l'OFPRA s'inscrit dans la continuité de l'orr, avec le même mode de fonctionnement, une division en sections nationales et une instance d'appel. De surcroît, les officiers de protection (nom emprunté à l'OIR) chargés d'instruire les demandes d'asile et d'assister les réfugiés sur le plan social et juridique, sont pour plus du tiers des anciens de l'orR et des Offices de réfugiés.

Le profil social des premiers officiers de protection de l'OFPRA marque également la singularité de cette institution par rapport aux autres administrations françaises. En 1956, seuls trois d'entre eux (sur 21) sont nés en France et tous les autres sont de la même nationalité que les requérants qu'ils reçoivent. Ce sont presque tous des hommes, d'âge mûr, et plus de la moitié sont eux-mêmes réfugiés ou apatrides. Socialement, ce sont des notables qui ont souvent été engagés dans la vie politique de leur pays avant de s'exiler en France. Le chef de la section ukrainienne est par exemple l'ancien ministre des Affaires étrangères de l'Ukraine indépendante; le chef de la section russe est le frère du ministre de l'Intérieur de la Russie de 1912 à 1915 et lui-même ancien député de la Douma, puis ambassadeur de Russie en France. Les officiers de protection de la section polonaise appartiennent à de grandes familles de l'aristocratie de leur pays, tout comme ceux des sections hongroise et roumaine. L'une des caractéristiques importantes de cette première génération d'officiers est le prestige qui découle de la position sociale qu'ils occupaient avant la migration. Ils font figure de représentants de gouvernements en exil chargés de reconnaître la qualité de réfugié à leurs compatriotes se déclarant persécutés par le régime auquel ils se sont eux-mêmes opposés ou qui les a parfois directement chassés du pouvoir. Ils donnent ainsi à l'OFPRA le statut d'une bureaucratie consulaire autonome, chargée d'accompagner les étrangers dépourvus de consulat. La seule exception à ce tableau concerne la section espagnole qui compte deux Français, signe de la suspicion que les pouvoirs publics de l'époque entretiennent à l'égard de ces exilés systématiquement associés à la mouvance communiste. Ces profils témoignent également d'une légitimité qui repose principalement sur la connaissance intime de la situation dans les pays d'origine, dans la continuité de celles façonnées au sein des anciens Offices de réfugiés. 
À partir des années 1960, le départ à la retraite des premiers officiers de protection de l'OFPRA et leur remplacement par des Français pourraient accréditer l'idée d'une "nationalisation» de l'institution ${ }^{4}$. En réalité, la logique de proximité qui prévalait depuis les débuts de l'Office entre les agents et leur public est maintenue: s'ils sont plus souvent français, les nouveaux officiers sont originaires des mêmes pays que leurs prédécesseurs et sont affectés à des sections qui correspondent à leur origine nationale; ils sont de surcroît liés aux anciens agents par des relations familiales ou communautaires, les recrutements étant construits sur le principe de l'interconnaissance. Les requérants, qui sont pour l'essentiel yougoslaves et espagnols (excepté en 1956 et 1957 avec l'arrivée de presque 10000 Hongrois), sont encore assez peu nombreux, car beaucoup, notamment parmi les Espagnols et les Portugais, préfèrent

70 résider en France au titre de l'immigration économique ${ }^{5}$.

\section{L'ENCASTREMENT DE L'ASILE}

DANS LE CHAMP ADMINISTRATIF

En avril 1967, la signature du protocole de New York met fin aux réserves limitant l'application de la convention de Genève aux victimes d'événements survenus avant 1951 en Europe. Cette extension, couplée à la dissociation progressive entre réfugiés et main-d'œuvre étrangère, se traduit par le développement d'un régime d'asile à deux vitesses, privilégié pour ceux qui viennent d'Amérique du Sud et du Sud-Est asiatique, et plus strict pour les autres exilés.

Le principe d'une proximité linguistique et culturelle entre officiers et candidats à l'asile est maintenu pour les nationalités qui bénéficient de cette présomption favorable. Au milieu des années 1980, la quasitotalité des agents de la division Amérique du Sud sont espagnols et sudaméricains. Il en est de même pour ceux de la division du Sud-Est asiatique presque tous originaires de la péninsule Indochinoise. Cette proximité, d'abord culturelle mais aussi sociale et politique, va de pair avec l'accueil privilégié que l'OFPRA réserve aux Indochinois: entre 1979 et 1985, ils bénéficient de taux d'accord oscillant entre 95 et $100 \%$. De tels chiffres sont en fait le produit d'une politique officieuse qui n'applique pas les mêmes critères de sélection selon l'origine des demandeurs: les exilés du

4. Gérard Noiriel, Réfugiés et Sans-papiers. La République face au droit d'asile XIXe-XXe siècle [1991], Hachette, 1998, p. 214-215.

5. Alexis Spire, Étrangers à la carte. L'administration en France (1945-1975), Grasset, 2005. 
Sud-Est asiatique sont quasi automatiquement reconnus réfugiés selon une logique basée davantage sur leur nationalité que sur la recherche de craintes éventuelles de persécution. À l'opposé, les Africains et «autres » Asiatiques, comme les Zaïrois ou les Sri-Lankais, doivent apporter la preuve qu'ils risquent d'être individuellement persécutés en cas de retour.

Sous couvert de dette envers l'ancienne colonie indochinoise - qui ne s'applique cependant pas à la même période aux anciennes possessions africaines -, il s'agit par cette politique d'accueil de discréditer le nouveau régime indochinois, communiste et vainqueur de la guerre de décolonisation. Les réfugiés du Sud-Est asiatique, souvent jeunes, sont également perçus comme susceptibles de compenser le vieillissement de la population française en lieu et place des immigrés venant d'Algérie et d'Afrique noire. Leur réputation de travailleurs dociles fait d'eux une main-d'œuvre idéale pour un certain nombre d'entreprises industrielles affectées par la suspension de l'immigration mais pas encore touchées par la crise. Par comparaison, aucun agent originaire du continent africain, ou asiatique (hors Indochine) n'est recruté dans les divisions qui reçoivent ces demandeurs: pas d'agents tamoul ou zaïrois pour s'occuper de ces deux groupes qui montent en puissance à l'OFPra. De même, tandis que les fraudes commises par les Zaïrois sont largement médiatisées, celles des Indochinois sont étouffées ${ }^{6}$. L'image du réfugié devient celle de boat people à qui le statut conventionnel est systématiquement reconnu, tandis que la figure du demandeur d'asile (le terme apparaît au début des années 1980) devient celle d'un débouté potentiel, souvent venu d'Afrique.

De 1984 à 1988, le nombre de demandes d'asile progresse de 21714 à 34352 , ce qui incite les pouvoirs publics à engager de nouveaux officiers de protection (dont le nombre passe d'une petite vingtaine à une quarantaine). Dans toutes les sections autres que celles d'Indochine et d'Amérique latine, le renouvellement des agents dessine les contours d'une institution qui commence à se rapprocher, dans son fonctionnement, des autres administrations. Le profil des nouvelles recrues se démarque des générations précédentes: plus jeunes, plus diplômés, moins expérimentés et plus nombreux à être d'origine française. Âgés de 28 à 44 ans, ils sont en majorité titulaires d'une licence (en sciences humaines ou en langues et plus rarement en droit); pour certains, ils ont déjà connu des expériences professionnelles allant de l'enseignement au journalisme en passant par les associations de défense des étrangers et les organisations internationales.

6. Karen Akoka, «L'archétype rêvé du réfugié», Plein Droit, n 90, 2011, p. 16. 
Cette nouvelle génération incarne le passage d'une légitimité reposant sur la connaissance intime d'une langue, d'un pays ou de l'expérience de l'exil, à une légitimité fondée sur la qualification par le diplôme.

À cette évolution des recrutements et de la composition des flux de demandeurs, plus souvent originaires du continent africain, correspond également un changement dans les pratiques des officiers de protection. La décennie 1980 est marquée par une inversion de tendances ${ }^{7}$ : en 1985 pour la première fois, l'OFPRA rejette plus de demandes qu'il n'en accepte $(56 \%)$. La proportion de reconnaissance, c'est-à-dire la part des reconnaissances parmi l'ensemble des décisions de l'année, passe de $85 \%$ en 1980 à $15 \%$ en 1990.

\section{L'ENRÔLEMENT DE L'OFPRA}

72 Dans L'objectif De maîtrise des flux migratoires

Suite à l'augmentation de la demande d'asile qui intervient entre 1988 et 1989 (de 34352 à 61 422), le gouvernement de Michel Rocard débloque d'importants moyens financiers et humains pour raccourcir les délais d'instruction. Le budget de l'office est multiplié par quatre en deux ans et le nouveau directeur procède à des recrutements massifs puis met en place une nouvelle organisation du travail.

L'informatisation et le pilotage par objectifs chiffrés se développent progressivement. Si ce mode de gestion s'impose dans un grand nombre d'autres administrations touchées par les réformes managériales, les exigences de rendement se traduisent à l'OFPRA par une multiplication des décisions de rejets sans entretiens ( $83 \%$ en 1990) et par une chute des proportions de reconnaissance à un niveau jusqu'alors inégalé (15\%). Parallèlement, tandis que les agents de l'ofPra étaient jusque-là exclusivement des contractuels, la création en 1991 du corps des officiers de protection leur permet de devenir fonctionnaires pour la quasi-totalité d'entre eux. Dans le même temps, une nouvelle division du travail voit le jour: les missions de protection sont dissociées des fonctions d'éligibilité alors qu'auparavant les deux étaient assumées par les mêmes agents. Ainsi, la dimension sociale du métier d'officier de protection s'efface au profit de la dimension de contrôle. Alors que, jusque-là, leur mission consistait à articuler intégration et sélection, ils ne s'occupent, pour la plupart, plus que de sélection et, dans ce cadre, ne rencontrent plus de réfugiés reconnus. Progressivement, les profils atypiques et les militants

7. Luc Legoux, La Crise de l'asile politique en France, Ceped, 1995. 
engagés disparaissent des fonctions d'encadrement. Désormais, tous les directeurs généraux sont énarques, alors qu'aucun d'eux ne l'était jusqu'à la fin des années $1980^{8}$.

Les années 1990 se caractérisent par une chute constante (hormis une très légère hausse en 1997 et 1998) du nombre de demandes d'asile qui s'explique par une politique rendant l'accès au territoire de plus en plus difficile. Par la suite, face à la nouvelle augmentation des demandes d'asile qui survient en 1999, l'OFPRA choisit de faire appel à un nombre important de contractuels (près de 190 entre 2000 et 2004). Puis, entre 2005 et 2008, certains sont titularisés et de nouveaux postes d'officiers de protection sont créés, réservés pour moitié à la voie externe. L'ensemble de ces arrivées modifie encore de façon importante le profil social des agents. Les officiers de protection sont désormais majoritairement issus des classes moyennes supérieures (très souvent enfants de cadres ou d'enseignants), ce qui contribue à creuser la distance sociale qui sépare les requérants de ceux qui les jugent. L'âge moyen au moment de l'entrée en fonction continue à baisser et le nombre d'agents recrutés après d'autres expériences professionnelles également. Parallèlement, le niveau de diplôme augmente: les trois quarts ont un double diplôme dont un de troisième cycle. Cette élévation des qualifications, que connaissent d'autres administrations, est renforcée à l'OFPRA par la création du corps des officiers de protection qui contribue à ennoblir le métier; les promotions du statut de secrétaire à celui d'officier, encore fréquentes dans les années 1980 et 1990, n'ont désormais plus cours. Les officiers de protection des années 2000 sont moins nombreux que leurs aînés à être issus de cursus en sciences humaines mais beaucoup plus fréquemment titulaires de diplômes de droit ou de langues et civilisations. Cette évolution dans les formations renforce le passage d'une vision politique de l'asile à une conception juridique et positiviste du réfugié, accréditant l'idée d'une procédure qui serait neutre et objective. La reconnaissance de la qualité de réfugié apparaît alors comme une épreuve de vérité 9 ou une enquête qu'il s'agit d'élucider par recoupement des sources et confrontation des éléments du récit avec un stock de connaissances considérées comme intangibles et «vraies». Un même rapport à la vérité structure la démarche des juristes ou des spécialistes de pays: qu'ils se réfèrent à un texte de droit ou à

8. Sur l'ennoblissement de la haute fonction publique en charge de l'immigration, voir Sylvain Laurens, Une politisation feutrée. Les hauts fonctionnaires et l'immigration en France (1962-1981), Belin, 2009.

9. Estelle d'Halluin-Mabillot, Les Épreuves de l'asile. Associations et réfugiés face aux politiques du soupçon, EHESS, 2012. 
une expertise géopolitique, ils conçoivent leur activité de juger comme un processus de dévoilement permettant de déterminer s'il s'agit d'un migrant ou d'un réfugié.

À cette évolution des profils des agents recrutés s'ajoutent d'importantes transformations dans l'organisation du travail. La généralisation d'un pilotage de l'activité par objectifs chiffrés se traduit par une évolution significative dans l'ordre des priorités bureaucratiques. Tous les jours, chacun comptabilise le nombre de dossiers qu'il a instruits. Les comptes sont remis mensuellement aux supérieurs hiérarchiques qui, selon les cas, réprimandent ou félicitent. Parallèlement des primes sont attribuées à ceux qui «font leur chiffre». Ce mode de fonctionnement renforce la dépendance de l'agent vis-à-vis de sa hiérarchie et du même coup le processus d'intériorisation de la contrainte hiérarchique. Elle influe 74 sur la nature des décisions prises. Quand un doute surgit à propos d'un dossier, il est plus commode de rejeter que de proposer une décision d'accord qui nécessite davantage de recherches et qui doit toujours être dûment argumentée auprès des supérieurs ${ }^{10}$. Pour beaucoup d'agents, la politique du chiffre se traduit donc par une situation paradoxale: alors qu'ils conçoivent l'acte de reconnaissance de la qualité de réfugié comme la quintessence de leur mission ( «quand on fait des accords, on a l'impression de faire notre métier»), ils en viennent à préférer les «mauvais dossiers» car ils sont plus faciles à instruire et permettent d'atteindre plus vite le chiffre. En revanche, les décisions de reconnaissance de la qualité de réfugié demandent des entretiens plus approfondis, et un travail documentaire poussé pour emporter la conviction du supérieur hiérarchique.

L'emprise des normes hiérarchiques devient plus prégnante encore dans les années 2000. Par exemple, les officiers de protection ne peuvent plus choisir leurs dossiers; ce sont leurs supérieurs qui les leur remettent, après avoir procédé à un premier examen et éventuellement ajouté des annotations sur les orientations à prendre. Il ne leur est également plus possible de recevoir directement des appels de bénévoles ou de salariés associatifs pour discuter des dossiers, ni d'interroger le centre de documentation ou celui des affaires juridiques, sans l'aval de la hiérarchie. Ce qui était considéré comme relevant des arbitrages personnels devient prescrit et contrôlé. La conséquence est la dilution du pouvoir de décision et la déresponsabilisation. Les officiers de protection s'apparentent

10. Alexis Spire, Accueillir ou Reconduire. Enquête sur les guichets de l'immigration, Raisons d'agir, 2008. 
désormais à un maillon d'une chaîne bureaucratique; ils ne sont plus confrontés à un cas dans sa globalité, mais interviennent davantage sur le segment d'une procédure qui s'étale sur plusieurs mois, voire plusieurs années.

Le processus d'assujettissement de l'action des agents de l'ofpra à la lutte contre la fraude et à la maîtrise des flux migratoires est parachevé en 2007, lorsque Nicolas Sarkozy, qui vient d'être élu président de la République, décide de rattacher l'office au tout nouveau ministère de l'Immigration et de l'Identité nationale. Ce réagencement bureaucratique se traduit notamment par l'allongement de la liste des pays considérés comme "sûrs ", c'est-à-dire dont les ressortissants sont soumis à une procédure accélérée: les demandeurs n'ont alors que huit jours (au lieu de vingt et un) pour préparer leur dossier et sont privés d'allocation d'attente. En outre, la suspicion que le pouvoir politique entretient à l'égard de ceux qu'il considère comme de «faux réfugiés » peut être directement relayée au sein même de l'institution chargée d'étudier les demandes. C'est notamment le cas en novembre 2011, lorsque le directeur de l'OFPRA enjoint à ses services de rejeter sans examen approfondi les demandes déposées par des étrangers ayant altéré leurs empruntes digitales.

Depuis la fin des années 1980, l'action des officiers de protection de l'OFPRA est encadrée par un nombre croissant de normes et d'instructions répondant davantage à l'objectif de maîtrise des flux migratoires qu'au souci de protéger les réfugiés. Ils ne sont plus qu'un acteur parmi beaucoup d'autres: les demandeurs d'asile doivent d'abord arriver à accéder au territoire, puis passer par la préfecture pour bénéficier d'un droit au séjour et après le passage par l'OFPRA, la quasi-totalité de ceux qui sont déboutés déposent un recours devant la Commission des recours des réfugiés, devenue en 2007 Cour nationale du droit d'asile. À cette complexité procédurale s'ajoute l'ambiguïté des chiffres qui nourrit de lancinants débats autour des statistiques de l'asile et de la position de la France par rapport à ses voisins. En effet, la France se présente comme le pays européen qui enregistre le nombre le plus élevé de demandes d'asile, mais, si on pondère ce nombre avec celui de la population du pays d'accueil, elle se classe au $12^{\mathrm{e}}$ rang sur $31^{11}$. De même, elle apparaît en $2^{\mathrm{e}}$ position si l'on considère le nombre total de décisions de protection (statut de réfugié et protection subsidiaire), mais tombe au $22^{\mathrm{e}}$ rang si l'on retient le critère du taux de reconnaissance.

11. Cf. UNHCR, Statistical Yearbook 2010,10édition, chap. 4. 
Le retour sur l'histoire sociale de l'Office français de protection des réfugiés et apatrides montre que l'évolution des conditions d'application du droit d'asile en France dépend non seulement des rapports de force politiques au sein de l'État mais aussi des logiques de recrutement qu'une telle institution poursuit au fil du temps. Jusqu'à la fin des années 1970, une sorte d'homologie est à l'œuvre entre, d'un côté, les officiers de protection, souvent anciens réfugiés, qui rédigent et parlent dans la langue des requérants et, de l'autre, des requérants qui sont proches d'eux culturellement et politiquement et qui sont considérés comme de futurs réfugiés. La latitude qui leur est laissée n'est néanmoins nullement le signe d'un laisser-faire. Elle s'explique en partie par le fait que les dispositions et les positions particulières de ces agents rencontrent les

76 intérêts des pouvoirs publics. On peut parler en ce sens d'une forme très contrôlée de non-contrôle ${ }^{12}$. Cette configuration perdure tant que ces réfugiés sont perçus comme utiles: ils permettent non seulement de décrédibiliser le système communiste mais aussi de valoriser la France comme terre d'accueil. À la fin des années 1980, l’objectif de maîtrise des flux migratoires l'emporte sur toute autre considération et l'OFPRA perd progressivement de son autonomie par rapport au champ bureaucratique chargé du contrôle des étrangers. Le traitement de l'asile passe ainsi de la subordination aux politiques diplomatiques, se traduisant par un fort taux d'accord, à un assujettissement aux politiques migratoires s'exprimant par un fort taux de rejet. Ce revirement prend forme dans une série de textes et d'instructions, en partie issus de règlements européens, mais il se manifeste également dans les conditions de recrutement et les nouvelles contraintes qui pèsent sur les agents chargés de les appliquer. C'est cette dimension pratique des politiques d'immigration que met en lumière l'histoire sociale de celles et ceux qui les ont mises en œuvre.

12. Rémi Lenoir, «Contrôle (du) social», Informations sociales, n 126, 6/2005, p. 6-15. 
Cet article propose d'appréhender l'asile politique à travers l'bistoire sociale des institutions et des agents qui l'ont mis en cuvre en France depuis la fin de la Seconde Guerre mondiale. Jusqu'à la fin des années 1970, les officiers de protection ont un profil social, culturel et politique très proche des réfugiés qu'ils reçoivent. Puis, au fil des recrutements et avec la généralisation de l'objectif de maitrise des flux migratoires, ils perdent de leur autonomie, an point de devenir un segment parmi d'autres de l'administration des étrangers. L'bistoire du rapport entre l'État et les étrangers peut ainsi se lire à travers la relation entre les priorités politiques des gouvernants et le profil social des agents recrutés pour les appliquer. 